\title{
Communication System Architecture for Planetary Exploration
}

\author{
Richard Alena. Bruce Gilbaugh, Brian Glass \\ NASA Ames Research Center \\ Molfett Fietd. CA 94035-10000 \\ 650-604-0262

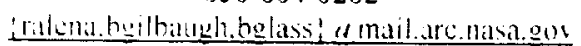 \\ Stephen P. Braham \\ Poly LAB. TIME Centre \\ Simon Fraser Lniversity at Harbour Centre \\ 515 West Hastings St. \\ Vancouver, BC, Canada \\ $604-268-7981$

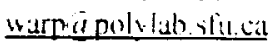

\begin{abstract}
fostract-Future human missions to Mars will require effective communications supporting exploration activities and scientilic lield data collection. Constraints on cost. size. weight and power consumption for all communications equipment make optimization of these systems very important. These intormation and communication systems connect people and systems together into coherent teams pertorming the difficult and hazardous tasks inherent in planetary exploration. The communication network supporting vehicle telemetry data. mission operations. and scientitic collaboration must have excellent reliability and tlexibility.
\end{abstract}

W: propose hybrid communication architectures consisting of space-based links, a surtace-based deployable mid-range communications network and a cluster of short-range links to solve the probiems of connectivity and bandwidth. while meeting the other constraints of weight and power. A network of orbiting satellites could cover much of the planet surface, but this space-based capability may not be optimal for cost or performance. Specifically. a minimal space-based capability can be augmented using mobile cellular repeaters deployable by robots and human EVA. This method results in an increase in the number of radio nodes, but the distances separating them is decreased. This results in a significant increase in bandwidth and decrease in radio power and therefore node size, complexity and power consumption. The paper will discuss the results of fieldtesting such hybrid radio systems for the support of scientitic surveys. System analysis of design tradeoffs will yield insight into optimal solutions that will be compared to other approaches providing a method of etfectively evaluating new candidate architectures.

Copyright 2001 by the Instituce of Electrical and Electronics Engineers. No copynght is asseried in the United States under Title 17. U.S. Code. The U.S. Govermment has royalty-iree license to exercise all nghts under the copynght claimed herein for govemmeni purposes. All ocher righis are reserved by the copynght owner.

\section{TABLE OF CONTENTS}

1. Introduction

2. Background

3. Hybrid Communications Architecture

4. Wireless Network Performance Measurements Link A vailability Bandwidth and Network Throughput Latency

5. Repeater Design Tradeoffs

6. MEX Communications System Example

7. MEX Field Test Results

8. Conclusions 
I. INTR()h) (CTHN

Reliathle communication iystems are essential for providing sallety and operational capahility for human planetary exploration missions. The reliability and cost factors of a communication system are tunctions of the architecture. In tiact. the archilecture of a sy stem determines yuite a range of properties for that system, such as redundancy. number of elements. connectivity and cost. This paper will explore hybrid architectures consisting of space-based elements and deployable ground-based elements. The proposed architectures will be evaluated for basic properties to help guide tuture technology development decisions.

The NASA Reference Mission to Mars will be used as a template for determining mission operational requirements relevant to the communication systems.' The Reference Mission baselines a small team of humans living on the planet surface in a habitat module as their exploration base, using resources provided by pre-deployed energy. oxygen and fuel production facilities. These human explorers would be assisted in their exploration traverses by a variety of robots. They would use small vehicles to pertorm short traverses and larger pressurized vehicles for longer traverses being away from the habitat for days at a time. They would use a variety of instruments both hand-held and vehiclemounted for operating support systems and gathering scientific data.

Communications technology is essential for the exploration of other planets. However. communications between instruments, robots. and eventually astronauts on the surface of a distant planet presents specific constraints that must be addressed by novel technical solutions. For instance. Mars presents varied terrain with substantial relief (craters. canyons, volcanoes. etc.). has no stable ionosphere to reliably support ground-based long-range wireless communications. The planet presents a hostile environment that requires high-speed video, audio and data communication to ensure safe robotic and human mission operations with the highest possible science return. In addition. the wide variation in expected communication performance, both for surface operations. and between Mars and Earth, leads to signiticant design issues. The facilities for this mission would consist of long-range space communications with one or more satellites in orbit around the remote planet augmented by ground-deployed facilities for local communication. An analysis of the performance benefits of various combinations of these elements would be very usetul for developing exploration scenarios more fully.

\section{BN(KIIRU)INO}

Nisil Ames Research Cinter is developing concepts and cechnology demonstrations for planctary surface exploration. The Wobile Exploration Sistem (WEX) project in the Computational Sciences Division. led by Rich Alena. is incegrating a varicty of digital multimedia capabilities for supporting remote tield science surveys and mission operations. These media include digital voice communications and recognition. pen-based display and human interation, digital video. high-resolution digital images and scientific instruments. vew efforts include space suit technology and robotic rover interactions. These elements are all linked using high-bandwidth wireless local area network (LAN) technology capable of covering several kilometers.

The MEX project works collaboratively with two other groups for exploration technology field tests. Simon Fraser University (SFU). and Canada ${ }^{3}$ Communications Research Centre (CRC) are examining the development needs for communication technologies that will enable future support of exploration missions to other planets. in particular to Mars." Advanced communications technology has alread? been experimentally deployed successfully during the 1999 and 2000 tield seasons of the N.ASA Haughton-Mars Project (HMP). a Mars analog field research program tocused on the Haughton impact crater site. The crater is located on Devon Island, Nunavut, in the Canadian High Arctic. During the 2000 field season of the H.MP. the implementation of satellite links to the Internet and deployment of three different regional networks strengthened the ongoing collaboration between NASA and Canadian research institutions and industr. The Telematics Research Laboratory at Simon Fraser Lniversity (SFC) worked with the SFU PolyLAB Advanced Collaborative Networking Laboratory to provide and manage space-based communications. a regional radio-based networking solution, and infrastructure integration. The MEX team deployed two other regional radio systems, linking allterrain vehicles (ATV) to base camp.

The NASA Haughton-ilars Project (HMP) is a NASA-led field research program (Principal Investigator Pascal Lee). dedicated to the study of the Haughton Crater and surroundings. ${ }^{3}$ The twenty-kilometer diameter crater was formed in an impact approximately twenty-three million years ago. The region is a cold, polar desert, and thus presents a wide variety of geological features and biological attributes that may shed new insights into the nature and evolution of Mars. The scientific study of the Haughton site provides a unique opportunity to also study the strategy: technologies, and human factors relevant to planning the future exploration of Mars by robots and humans. 


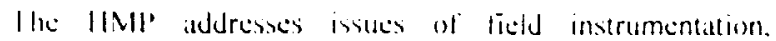
trallsportation, communications, and team-based scientific research in a relatively hostile and isolated environment. This includes the operation of robotic elements in the lield and their synergy with human exploration. expecially with regards to communications. From the standpoint of communications. the topography at Haughton tan be characterized as presenting an intricate network of hills and valleys. including vast tracks of inter-valley rocky plateau surfaces and deep canyon systems. The region experiences periods of extreme cold $(--5)^{\circ} \mathrm{C}$ in winter: $-0^{\circ} \mathrm{C}$ in summerl. and transportation of hardware systems across the region by all-terrain vehicles and autonomous robots intlicts high impat and vibration lodeds on electronic equipment. Furthermore. logistical support available at the site is limited. and the science and exploration research teams in the tield are taced with the challenge of having to setup. configure. monitor, and maintain complex systems to ensure that high-quality science intormation is collected while coping with field survival and safety concerns. This activity has vielded important insights into communication system architecture. $^{4}$

The MEX is used to develop operational scenarios and tools for collaborative mobile computing. The tield exploration environment poses signiticant constraints on the interaction of humans with data system components. The humans are hampered by their clothing which protects them against the cold windy environment restricting their movement and vision: they must use their hands to drive the vehicles. run instruments and take notes. Data system design needs to accommodate these differences while providing the situational awareness and science support required for safe productive exploration.

The MEX project is an outgrowth of previous work on wireless networking and collaborative sottware tools for Space Station." The MEX uses vehicle-mounted and handheld video cameras to provide viewpoints to remote observers and collaborators. Streaming digital video from these cameras provides continuous high-band width data that allows stress testing of the wireless network links, providing a high-level measure of link performance. Additional MEX iunctions include digital still cameras for detailed records of samples and sites and mapping capability based on Global Positioning System (GPS) to provide a real-time track of movement and position. A variety of communication modes between the field party and base camp are provided including voice channels and e-mail. Collaborative software allows creating pen drawings on shared white boards for diagrams.

\section{HYBRID COMMI VIC AIIONS ARCHIECTL RE}

We advocale a hybrid architecture consisting of a small number of satcllites around the planet (1) provide adequate relay capability to the plantary work sites from earth and mobile deployable ground-based repeaters to provide highbandwidth low-latency communieations between base and remote field parties. Each mobile repeater provides communications to a cluster of instruments and computers located within its cell. as well as providing backbone communication between repealers. This architecture provides optimal pertormance while minimizing costs of deploying the communication intrastructure.

On one end of the spectrum of architectural choices. purely space-based communication systems can be used. Each planetary node would have a satellite ground station for communications. The satellites could be sunchronous with respect to locations on the planet surface or could form a varing constellation in low orbit. ${ }^{7}$ This is equivalent to geo-synchronous (GEO) or low-earth orbit (LEO) methods used today. Iridium and other ventures have deployed LEO constellations for phone contacts anywhere in the world. However. the dominance of cellular phone systems suggests that perhaps ground-based repeater systems could be more cost etfective for local links.

The basic problem with space-based communications is that the distances involved require ground transceivers to be more powerful. with larger antennas to provide the gain required. These communication distances can be anywhere from 100 miles (LEO) to 24.000 miles (GEO) for earth. For a given receiver sensitivity. Eltective Radiated Power (ERP) scales as the square of distance. Theretore ground-based systems have an advantage of requiring about $10^{3} \cdot 10^{3}$ times less power (30-80 dB) per link than space-based systems. These advantages also apply to using smaller antennas that do not need precise pointing, or even omni directional antennas resulting in small compact transceivers. Batteries and power sources scale similarly. However, more repeaters are required for adequate coverage. Anyone with a miniature cell phone can attest to these advantages.

This hybrid architecture maximizes bandwidth for local data communications while minimizing distance between planetary elements, which results in minimum size weight and power consumption for the communication components. Latency would be very low. allowing real-time monitoring and remote control over the links. with very high-bandwidth accommodating multiple video and data channels on each link. Additionally, the repeaters in the system can be used tor other purposes such as differential position determination and environmental sensors. 
The use of inohile repeaters, ether mounted on ATVS ridelen by humans or mounted on rohots that can be sent to optimal locations is a hey concept for a deployable wireless regional network providing high-handwidth connections to remoti arcis. Mobile repeaters allow dynamic ehanges to nctwork copology supporting a much greater operational area and can accommodate the constraints of rugged terrain. They can be deployed to cover a given survey site and then re-deploved to cover another site minimizing the total number of repeaters needed. Each repeater can host a local cluster of wireless instruments and computers providing significant operational llexibility and capability.

There may be more than one such deployable cluster. Field parties more than $10 \mathrm{~km}$ away from base would require the use of a pressurized vehicle anyway, and this vehicle would carry a ground station for the satellite link, becoming a temporary base. Mobile repeaters deployed from the pressurized vehicle would provide communication with exploration parties going out for a day. In this manner. multiple clusters could support complex exploration missions with the space-based links providing the backbone between the clusters.

\section{Wireless NetWork PERForMaNCE MEASLREMENTS}

Pertormance can be characterized by several Quality of Service (QoS) parameters:

- Link Availability

- Bandwidth and Network Throughput

- Latency

\section{Link frailatilin}

The single most important QoS parameter may be link availability or connectivity. Link availability is simply the probability that the link is able to pass data at any given time. Connectivity is a complex function of radio frequency and design. terrain and electromagnetic propagation characteristics. On earth, radio frequency is very important since low frequencies bounce off the ionosphere and therefore are not limited to line-of-sight. However, many planets do not have ionospheres.

Digital radio communication systems are required because of the high data rate needed for communications with scientists in the field ranging from audio and video to science data and control messages. These systems operate at ultra-high frequencies (UHF) to maintain correspondingly high bandwidth. and thus are generally blocked by hills and canyons, producing serious signal fade. Furthermore, these hills reflect radio waves, causing interference between signals travelling along multiple paths. Fade at highfrequencies requires the deployment of radio repeater yitem, througheut the arcia, in particular on various high points whth gend line of sight to science research sites. lhese repeater shitems must be monitored and maintained in the ticld. which poses important logistical constraints of relevance to ruture exploration missions. The number of repeiters necded is dependent upen the terrain.

There are properties associated with the physical laver (radio transceivers) of wireless networks such as range. coverage and modulation rate. The range is the distance the radio link can transmit data. the coverage is the direction and area in which reception can occur. and tinally the modulation rate is the raw data bandwidth carried bs the radio signal. Modulation rate and data rate are relatej. but may not be the same due to encoding schemes

All communication links vary in pertormance versus time. The received signal strength (RSSI) of a link can vary widely. In space communications, particularly trctic communications. scattering and refraction of the radio signals as they pass through the ionosphere and troposphere respectively, can produce constantly changing RSSI. The regional network links suffer similar phenomenon due to changing antenna configurations on the mobile elements. Interference between radio elements can cause performance degradation.

\section{Bundwidth and Venvork Throughput}

Bandwidth determines the amount and form of data that may be delivered or returned for an exploration tield site. $A$ low-bandwidth link generally requires less power. and may be suitable for telemetry requirements. A high-bandwidth link. however, will be required when live video and large amounts of imaging data must be returned from the field site. Elfective Radiative Power (ERP) requirements for links are roughly proportional to bandwidth. both over spacebased and land-based communications links.

A high-level measure of effective bandwidth is network throughput expressed in packets per second, bits per second or bytes per second. A network monitor will provide such measurements directly and can be placed at various points in a system to help pinpoint bottlenecks. Throughput is a complex interaction of physical (radio) layer performance. network protocols and application layer. Monte Carlo methods can be used to simulate and determine performance, but MEX uses direct network monitoring.

Interference between different radio systems is a significant design issue that limits the choice of frequencies and placement of equipment. The primary carrier frequencies of each link segment can interfere with each other directly. This is worst when the beam of one system impinges upon the antenna of another system that uses similar frequency bands. Generally, the more sensitive receivers are most 
aflected. Correct plamming and placement of antennas is the primary solution. Lise of differene frequency bands for dillerell linh:s can also be dflective. Interference can result in low throughput or poor linh atvalability

lanenty

The latency or time lag present in a communications link determines how it may be used in human and robotic missions and is generally proportional to the distance between the elements. In surface to orbit communications. of the kind that may be used to extend communications across a large region on Mars. latency is on the order of milliseconds and is due mostly to buffering of data in repeaters. Humans on a planetary exploration mission will be in close collaborative communication with their local base, and an intrinsic part of the communication loop. Interplanetary communications require significantly different solutions. With propagation delays reaching up to forty minutes for an Earth-Mars link. long delays are introduced for direct interaction. These long delays will require different methods of interaction not dependent upon real-time response and will have a signiticant effect on the way missions will be run.

\section{REPEATER DESIGN TRADEOFFS}

Complex design tradeoffs are involved for the development of communications systems for exploration. Lnderstanding the variables and their interaction allows making intelligent choices. Designing robust communication systems that meet the constraints of space operation requires analyzing the real requirements of such systems and using effective architectures that result in structures that have properties appropriate for the intended application.

The HMP tieldwork allowed understanding these relationships because the site is large. about $20 \mathrm{~km}$ in diameter, and the terrain is rough, with high hills and valleys covering virtually the entire area. There is no single point that is so high as to provide an optimal primary repeater site. The science survey sites are widely scaltered in a variety of directions from base camp. The environmental conditions are challenging for both humans and machines. These constraints help simulate the conditions anticipated for planetary exploration.

The rough terrain requires the use of many repeaters to clear the many hills between base camp and the survey site. The primary repeater site is chosen to provide a good link to base and a high point within line-of-sight of many of the proposed survey sites and/or mobile repeater sites. Proper advanced planning and analysis of field operations is required tor these reasons. In lact, computer terrain mapping programs can be used to determine optimal repeater sites by calculating line-of-sight restrictions. Since directional inlemmas are uned. ralallors are repuired to point them properly.

There are two hasic lypes of repeaters: in-band repeating and multi-band repeating. The in-band type incorporates just one radie transceiver and a single antenna, otien omni directional. It takes a packet from the source, butfers it internally, and then repeats it after receiving it. Since the single transceiver cannot simultaneously transmit and reccive. it imposes a 50$)^{\circ}$ o duty eycle on the throughput. Therefore each repeater hop can result in a $500^{\circ}$ o reduction in raw bandwidth! Pipelining can reduce this for multiple hops. but the performance impact is still signiticant.

The multi-band type uses two radio transceivers working on slightly different frequency bands and feeding two separate antennas. These repeaters can simultaneously receive a packet and re-transmit it on the other channel. This should theoretically result in more bandwidth when multiple hops are involved. Multi-band repeaters use two antennas. which can be pointed in different directions. solving interference problems while providing gain for additional range.

In-band repeaters have the advantage of simplicity, while multi-band repeaters have the performance advantage. We advocate a simple in-band low-bandwidth omni-directional telemetry system in conjunction with a multi-band highbandwidth directional system to take advantage of both approaches. A single in-band radio is combined with two multi-band radios to produce this hybrid. The resulting system features redundancy to accommodate component failure since telemetry can be carried on the high-rate link should the low-rate link fail. This dual-mode model has been used for spacecratt communication systems for many years.

The following diagram shows a representative repeater design with these characteristics. It combines a lowbandwidth omni directional radio with two high-bandwidth radios feeding two directional antennas acting as a multiband repeater. The directional antennas use rotators for pointing. The high-bandwidth repeater is used for LAN connections while the low-bandwidth system is used for control and voice communications. A processor is included allowing crossover of data to the high-bandwidth system as a substitute for the low-bandwidth system. This processor could also determine time delays in communication pulses allowing determination of position differentials. In the event of high-bandwidth system failure, there is still basic control. telemetry and voice communications through the lowbandwidth system. This approach is single-fault tolerant. providing basic communication capability and monitoring for safety. 


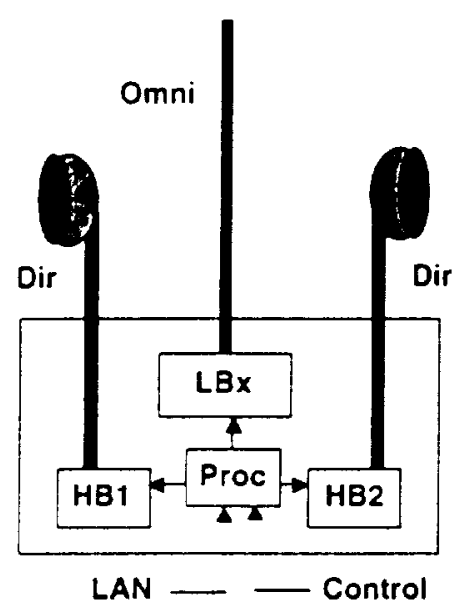

Figure 1. Repeater Conliguration

Cost is a function of the combined requirements for power, size, volume. weight. component selection and number of elements. The basic requirements for the design of deployable communication systems requires understanding the extent of the area to be covered. the terrain involved and the constraints on repeater placement. For example. one can trade off radio power and antenna complexity against the number of repeaters required. The repeater package described above san be made very compact and reliable. even using today's technology. For a ground-based system covering distances of $3-20 \mathrm{Km}$. the radio power is on the order of a watt apiece. and the total repeater power consumption could be well under 5 watts total for bandwidth in the mega-bits per second.

There are other tradeotfs for repeater design worth mentioning brietly. Bandwidth scales directly with effective radiated power (ERP). Line-of sight range scales directly with ERP squared. ERP can be increased by using high-gain directional antennas, but at the expense of coverage. The bandwidth, range and coverage requirements come directly from the anticipated mission scenarios. Therefore defining constraints on the range and direction of planetary exploration traverses due to life support, vehicle and safety concerns is the tirst step.

Another major tradeorf involves the frequencies used for communication. Component and antenna size scales inversely with frequency, becoming much smaller as the frequency increases. Increasing frequency generally reduces required ERP for a given bandwidth. determined by noise floor. saving power. However, component cost and complexity increase with trequency so there is usually an optimal frequency band that is supported with robust, lowcost components. Currently, $2.4 \mathrm{GHz}$ components offer good performance at low-cost. Next generation Instrumentation Scientific and Medical band components will operate at $5.8 \mathrm{GHz}$ offering certain advantages.

\section{MEX COMMUNICATIONS SYSTEM EXIMPIE}

The MEX project uses rapid prototyping lechniques to allow early evaluation of technology in an analog field environment simulating planetary exploration. The MEX provides a framework for integrating a wide variety of devices and tools into a coherent Intranet of distributed elements. Sume of the elements are earth-bound. others are space-based resources and others are planet-based. We can analyze the computing and communication needs from a mission requirements perspective and propose architectures that deliver this pertormance at the lowest cost.

The following diagram shows the MEX communications architecture including the space and regional links provided by SFU and CRC. This architecture provides Internet connectivity down to the hand-held graphics tablets that the explorer uses to record field data. The HMP communications infrastructure provided a single unified TCP/LP subnet for all computers located at base camp and for all the MEX elements. This allowed all elements to talk to each other directly using TCPIP protocols. The router was at the satellite ground station. which projected the subnet through the satellite link at $38+\mathrm{Kbps}$. Other contigurations are possible. for larger networks a router at base camp would allow multiple subnets to be established in the field supporting more elements and providing some partitioning between users.

Experience with the Haughton site allowed the MEX project to determine certain numbers for the design of the communication systems. The total range needed to be about $10 \mathrm{~km}$. the longest single day traverse distance and the radius of the crater area. The distance to repeater sites was about $3 \mathrm{~km}$ maximum due to the terrain. Therefore two repeaters would be needed to cover the $10-\mathrm{km}$ maximum range. We wished to provide about 200-400 kbps of sustainable bandwidth. More bandwidth was desirable, but those radio systems cost more and drew much more power. Therefore, radio selection was based on cost, size, power consumption, and licensing issues to provide the basic performance outlined above. 


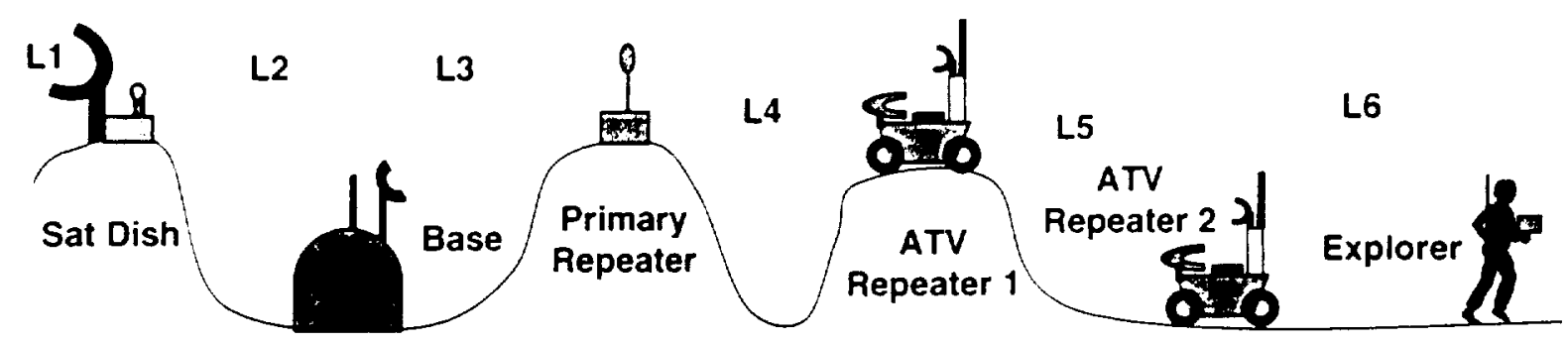

Figure 2. MEX Communications Architecture

We can calculate the number of repeater sites required and their approximate placement using Haughton crater as an example. Selection of base is made on a variety of criterion such as proximity to sites of scientific interest, exposure, resources and accessibility. It is not made on the basis of suitability for communications. However. to ensure contact with the space link, the primary spacebased ground station was located on a high hill and connected to the local base using a wireless link. Note that this hybrid has an immediate benetit of providing tlexibitity for the base location. by decoupling the base selection from the communications constraints. The only constraint is that there be a clear line-ot-sight between base and the satellite communications site to minimize the number of repeaters and maximize the reliability of this critical primary link. The satellite communications site should be within an easy traverse of base to simplify logistics.

The satellite dish for HMP near base camp to provide a clear view of the Anik-El satellite near the horizon represented as link LI in Figure 2. A WiLAN link was used to provide a link of + Mbps between the satellite dish and the base camp lab tent. ${ }^{\text {s }}$ The base camp WiLAN link (L2) provided Internet access for about 20 people working on laptop computers for science and mission simulations using 10BaseT Ethernet with multiple network hubs. The Internet connection also provided voice links to the mainland telephone system using Internet phone applications. The base camp lab tent was the equivalent of the local base for MEX operations during HMP2000.

MEX used both $900 \mathrm{MHz}$ and $2.4 \mathrm{GHz}$ Instrumentation Scientific and Measurement (ISM) band packet radio systems. The $900 \mathrm{MHz}$ Freewave system provided up to 115 Kbps over a serial connection and used omni directional antennas." The $2.4 \mathrm{GHz}$ Proxim system provided $1.6 \mathrm{Mbps}$ raw data rate using frequency hopping and a 10BaseT wired network interface. ${ }^{10}$ The $900 \mathrm{MHz}$ system provided somewhat greater range and connectivity than the $2.4 \mathrm{GHz}$ system, but at the expense of bandwidth. We performed various tests of range, bandwidth and connectivity using these radio systems to communicate between base camp and an ATV during HMP 99 and
HMP20)(0). These links have working ranges of several kilometers dependant upon antenna contiguration. The 2.1 $\mathrm{GHz}$ systems need to use directional antennas to provide the gain required tor this distance.

The MEX links used a central repeater located on a high hill about one kilometer from base camp. This location had excellent line-of-sight to most areas intended for communications testing and scientific surveys. In fact. choice of this primary repeater site is critical to link coverage and performance. The link between base and the primary fixed-site repeater is L3. The primary repeater site provides the height needed to reach other potential repeater sites. The mobile repeater is parked at one of these sites and relays the signal to the next mobile repeater site. Which finally relays the signal down to the survey site. which may be in a valley represented by L4 and $\dot{L} 5$. This results in three hops for routing data between base camp and the survey site. Given the number ot hops. the characteristics of the repeaters are critical for providing good end-to-end performance.

The MEX ATV had a repeater set up on it with antennas mounted on an elevated platform at the rear of the vehicle as shown in the following picture. This repeater could be used for linking various hand-held cameras and instruments to the regional network. Additionally. this ATV-mounted repeater could act as a mobile repeater for extending the range of the regional network. especially to areas that are out of sight of the primary repeater.

The first mobile repeater connected to the primary fixedsite repeater. It had to be within $3 \mathrm{~km}$ of the primary site. This covered an area of $28 \mathrm{sq} \mathrm{km}$ around the primary repeater. The use of sector directional antennas with a 110 degree horizontal radiation pattern allowed the antenna to cover a large area of the anticipated survey zone This resulted in nearly continuous contact, except when behind an obstruction. Each ATV could carty another repeater, so the second ATV would be placed on a hill about $3 \mathrm{~km}$ from the first ATV, resulting in $9 \mathrm{~km}$ from base camp and covering an area of $254 \mathrm{sq} \mathrm{km}$. Each repeater draws 4 watts with a radio power of $150 \mathrm{~mW}$. Antenna gain is about $6 \mathrm{~dB}$ on each end resulting in an ERP of $1.2 \mathrm{~W}$ for 
sich limh. Sin we had thres repeillers and ewa stations f balse and remote) bor a lotal power comsumption of anly to wills.

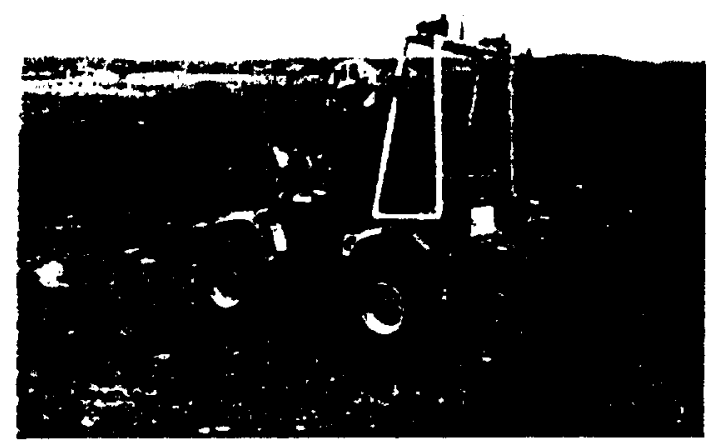

Figure 3. MEX ATV Configuration

\section{MEX FIII.1) TISI RHSI I}

The MEX rield-cested the riddie linhs determining connectivity and using network throughput as the primary measure. The effects of different antenna contigurations and interfierence between radio systems could also be determined. Some interesting results were obtained. most in line with expectations."

The satellite link provided 384 Kbps of data bandwidth. Lising downloads from the Internet as test tiles. this bandwidth was realized on a sustainable basis. These downloads used links $L 1$ and $L 2$. The only reliability issues were caused by moisture getting into the satellite dish waveguide and interterence to L2 from the other 2.4 Ghz systems, which resulted in packet loss. Continuous monitoring of the $\mathrm{L} 2$ link provided detailed data on the rate of packet loss and adjustments to antenna placement and turming off the other radio systems when they were not in use mitigated the problem.

The L3, L4, L5 and L6 links were all Proxim $2.4 \mathrm{GHz}$ links with identical performance. but with different antenna selections optimized for intended usage. The links pertormed retiably and well provided care was taken in pointing the antennas. Sometimes when the ATV was near base and in the line of sight between the repeater and base. it would lock onto the base camp link and not use the repeater. Such roaming is actually a feature of cellular systems. but create uncertainties for detailed measurements. Each of these links was monitored for RSSI as well as network throughput. With good sight lines. about $20 \mathrm{~KB}$ per second was realized on a sustainable basis. This corresponds to about $160 \mathrm{Kbps}$ end-to-end through all the links. Since only one ATV was outtitted during HMP 2000. L5 did not exist in the tield contiguration.

The $160 \mathrm{Kbps}$ compares favorably with the $240 \mathrm{Kbps}$ that such a multi-repeater link provides under optimal conditions. The link was robust when line-of-sight was maintained, but would degrade to about $16 \mathrm{Kbps}$ before the connection was lost. The only real problems noted in field-testing were that careful pointing of the antennas was required and that the radio gear needed to be kept very dry. There were also variations in RSSI that were not understood. We will improve the environmental protection and antenna pointing systems. Adequate performance of the video links and other collaborative applications was noted when the links operated at the full bandwidth. These applications operated badly under degraded link conditions sutfering very low frame rates and long latencies due to high packet loss. The batteries and solar power systems used were adequate for the task.

The $900 \mathrm{MHz}$ systems exhibited much higher connectivity during field tests conducted during HMP 99. 
Nthough limiled to line-of-cight. their higher power and lower handwath improved reception in marginal conditions. Connections were lost only when deep in cianyons and draws. Marginal conncctions at 20 Kbps were muintained even when slightly out of sight of the repeaters. Omni directional multi-element antennas with hdlB of gain were used for this system. We obtained connections at $8 \mathrm{~km}$ from base using direct line of sight. Even lower frequencies would allow better reception due to ditfraction. but tests with VHFF and $C B$ voice communication systems found that the advantages were not observable. This was due to the use of much shorter than optimal antennas for these lower frequency handheld radios, a common practice. Theoretically, a VHF data radio could provide tens of kilobits per second with a few watts of power and get around hills, but lield tests have indicated that much more reliable communication is attained using repeaters. Theretore, we do not believe that adequate data communications performance will be delivered by low frequency carrier systems, and the resulting components would be large in any case.

\section{CONCLUSIONS}

The hubrid architecture proposed delivered excellent capability in the lield, incorporating the best of both space-based and ground-based links. The space link was required for the long haul. but once a good link was established with base. the ground-based links provided very flexible and reliable performance. Management of the ground-based resources is a significant logistical challenge.

Wobile repeaters have advantages of providing extremely low-latency high-bandwidth pertormance using very linle power in a compact package. Satellite altematives would be much more expensive. requiring many satellites for adequate coverage and larger receivers on the surface for comparable pertormance. An optimal mix of satellite and ground-based elements could maximize performance and minimize cost. A detailed quantitative analysis of such a hybrid would be useful, but is beyond the scope of the present effort.

The mobile repeaters can be mounted on robots or vehicles or can be deployed in small packages for fixed sites. Therefore, the incremental cost of the repeaters may not be prohibitive, since the major resources would be in place. There are other advantages such as differential positioning, supply caching and environmental sensing that also favor deployable communication elements. Future development of MEX is anticipated for subsequent field deployment during scientific surveys and mission simulation activities. Full integration with science and operations teams will allow the collaborative capabilities of MEX technology to be demonstrated.

\section{Acknowledgments}

The authors would like to thank the NASA-Ames Haughton-Wars Project for the opportunity to participate and for the support provided by Dr. Pascal Lee (principal investigator) and Dr. Kelly Snook (project manager) who together led the expedition for HMP 2000).

\section{REFERENCES}

[1] VASA Mars Reterence Mission. NASA 1999

[2] Planet Net Project Site, URL

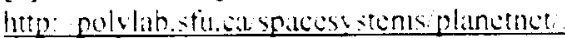

[3] Stephen Braham, Peter Anderson, Pascal Lee. Richard Alena. Brian Glass, "Canada and Analogue Sites for Mars Pixplorution," Proceedings of the Second Canadian Space Exploration Workshop. 1999.

[4] Stephen P. Braham, Richard Alena. Bruce Gilbaugh, Brian Glass (2001) Space . Vetworking and Protocols for Planetary Exploration and thalog Planetary Sites. IEEE Aerospace Conterence

[5] R. Alena (1996). Wireless Verwork Experiment - Risk Mitigation Experiment 1306. report on STS-74/76-Mir20 experiment for the Phase One International Space Station Program.

[6] R. Alena. E. Yaprak. S. Lamouri (1999), . Hodeling At Wireless Serwork For International Space Station. IEEE Aerospace Conterence.

[7] Interplanetary Internet Site. URL hur: "uwll innsigord.

[8] http:/www.wilan.com

[9] http://www. freewave.com

[10] Proxim Corporation. RangeLAN2 Technical Reference Guide. 2000. Proxim. Inc. USA. hup www grosim com

[11] Bruce L. Gilbaugh, Brian Glass, Richard Alena (2001). Wobile Nework Field Testing at H.MP-200n, IEEE Acrospace Conference 
10. BIOGRAHIIILS

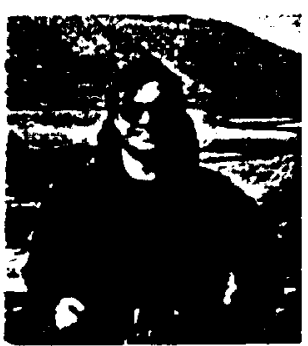

Richard Nena is a Computer Engineer and the Group L.eid fior the Intelligent Mobile Technologies (IMT) Lab and the Wohile Exploration System testbed at NASA Ames Rescarch Center. The IMT team integrates mobile hardware and soltware components into unique systems supporting human performance for flight and payload operations aboard spacecraft. $\mathrm{He}$ was principal investigator for the Wireless Network Experiment tlown aboard Shuttle and Mir. technology later adopted by the International Space Station Program. Rick spent three summers in the Canadian Arctic developing mobile technolugies for human planetary exploration. He has a WSEESCS from Cniversity of California Berkeley

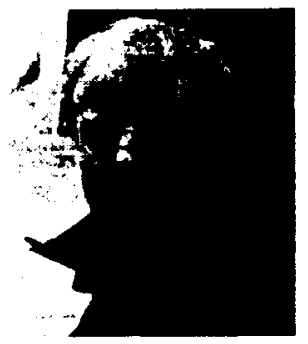

Stephen Braham is an Adjunct Professor of Communication. and the Director of the PolyLAB Advanced Collaborative Networking Laboratory at Simon Fraser Lniversity. Steve was instrumental in setting up the space-based and medium-range communications network for the 1999 and 2000 field seasons of the Haughton-Mars Project. He also participated in spacebased and wireless networked communication of physiolugical data obtained from climbers on Mt. MiKinley. AK. in May 2000, with a team from the Environmental and Aerospace Physiology laboratory at SFU. Steve is an experienced theoretical astrophysicist, and has become a specialist in the needs of advanced scientitic communication and exploration. Steve is also a co-founder and current President of the North American OpenMath Initiative (NAOMI). NAOMI is a collaboration between North American universities and industry to build a standard for communicating complex scientitis dats that includes crucial mathematical information.

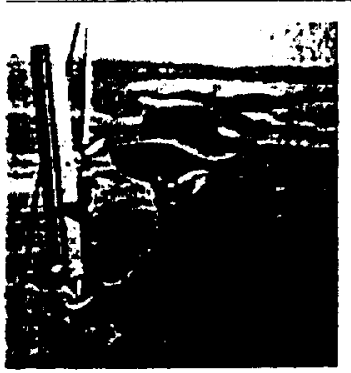

Bruce (jilbaugh is a member of the Intelligent Wobile Fechnologies (IMT) Group working on the Mobile Exploration Sistem (MEX) project at VASA Ames Research Center. He designed the regional wireless network radio configuration and the hardware mounting and power implementation on the All-Terrain-Vehicle for WEX testing during Haughton-Mars Project 2000 . He holds an AS in RF Communications Systems and Computer Networks, a BS in Information System Management from the University of San Francisco and is currently working on an MS in Telecommunications Wanagement at Golden Gate University.

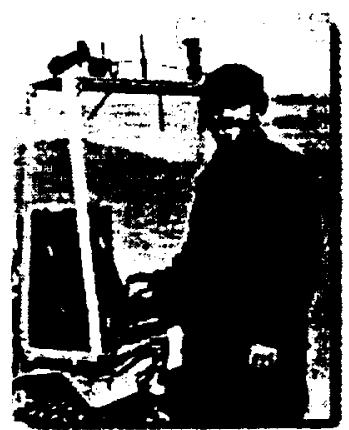

Brian Glass has been on the staff of NASA-Ames Research Center since 1987. He has led projects in automated reasoning (for Space Station and SETI health management). surface air traffic control (the Surface Movement Advisor, for which he shares a patent), and research groups in intelligent, adaptive control systems. In addition to his education and interests in engineering and computer science. he acquired an additional graduate degree in geophysics in 1992 focused on morphological models of scurp-like landforms. Brian is currently responsible for information and automation technologies in the Human Exploration Office and works closely with the Center for Mars Exploration. Over the past two summers. Brian has conducted field experiments at terrestrial analog sites in the Arctic to test human-centered computing concepts for future Mars geological exploration. 